\title{
La escucha de la tortura desde el Estado: la experiencia de los profesionales de la Comisión Nacional sobre Prisión Política y Tortura en Chile*
}

\section{The State's Listening of Torture: The Experience of Professionals in the Chilean National Commission on Political Imprisonment and Torture}

Recibido: noviembre 13 de 2011 | Revisado: mayo 5 de 2012 | Aceptado: junio 3 de 2012

\author{
Marcela Cornejo ** \\ GERMÁN MORALES *** \\ Pontificia Universidad Católica de Chile, Chile \\ JUANA KOVALSKYS ***** \\ Universidad de Chile, Chile \\ DARIELA SHARIM****** \\ Pontificia Universidad Católica de Chile, Chile
}

\section{SICI: 1657-9267(201303)12:1<271:ETECNP>2.0.TX;2-E}

Para citar este artículo: Cornejo, M., Morales, G., Kovalskys, J. \& Sharim, D. (2013). La escucha de la tortura desde el Estado: la experiencia de los profesionales de la Comisión Nacional sobre Prisión Política y Tortura en Chile. Universitas Psychologica, 12(1), 271-284.

* Artículo de investigación. Los autores agradecen a los profesionales de la CNPPT que participaron muy comprometidamente en esta investigación. También a todos los miembros del equipo de investigación por sus aportes valiosos en todo el proceso investigativo: Rodrigo Rojas, María Ester Buzzoni, Francisca Mendoza, Jenny Thiemer, Ximena Faúndez, Mariela Concha, Catalina Cabach y María Jesús Fontecilla. Este trabajo ha recibido financiamiento del Proyecto FOND. ECYT №1070855 "Del testimonio al relato de vida: procesos elaborativos en profesionales de la Comisión Nacional de Prisión Política y Tortura".

* Profesora Asociada. Escuela de Psicología. Avda. Vicuña Mackenna 4860 Macul, Santiago de Chile. E-mail:marcela@uc.cl,

**** Profesor Adjunto. Escuela de Psicología. E-mail: gpmorale@uc.cl

****** Directora Instituto Latinoamericano de Derechos Humanos y Salud Mental. Profesora Departamento de Psicología.E-mail: jkovalskys@mi.cl

****** Profesora Asociada, Escuela de Psicología. E-mail: dsharim@uc.cl

\section{RES U MEN}

Se presenta una investigación cualitativa cuyo objetivo fue comprender el impacto que tuvo escuchar el testimonio de víctimas de tortura sobre los profesionales que trabajaron en la Comisión Nacional sobre Prisión Política y Tortura, realizada en Chile entre 2003 y 2004. Se realizaron relatos de vida con 22 profesionales que trabajaron en esta Comisión, a partir de tres encuentros con cada uno de ellos. Los resultados muestran que el impacto de esta experiencia articula procesos elaborativos desde diferentes dimensiones -emocional, biográfica-narrativa e institucional- entendidas como coordenadas por donde circulan estos procesos, los que se revelan como profundamente influidos por las significaciones y sentidos que los profesionales construyen acerca de sus historias personales y familiares y de su historia social. Palabras clave autores

Comisión Nacional sobre Prisión Política y Tortura, elaboración psíquica, impacto emocional, tortura.

Palabras clave descriptores

Psicología Social y Política, enfoque biográfico, Chile.

\section{A B S T R A C T}

This article presents a qualitative study aimed at assessing the impact of the oral testimonies of torture victims on professionals who worked for the Chilean National Commission on Political Imprisonment and Torture in 2003-2004. Life stories were developed with 22 professionals who worked for this Commission based on 3 meetings with each of them. The results reveal that the impact of listening to torture testimonies articulates elaborative processes from different dimensions -emotional, biographical-narrative and institutional-regarded as a background of coordinates which frame their motion. Such processes are heavily influenced by the meanings and senses that the professionals construct about their personal and familial histories and about their social history. Key words authors

National Commission on Political Imprisonment and Torture, working through, emotional impact, torture.

Key words plus

Social and Political Psychology, Biographical Approach, Chile. 
Las políticas de reparación implementadas progresivamente por el Estado chileno durante los últimos 20 años para abordar las violaciones de los derechos humanos cometidas en Chile durante la dictadura militar entre los años 1973-1989, han buscado establecer la verdad de lo ocurrido y encontrar formas de reparación desde el Estado hacia las víctimas.

La Comisión Nacional sobre Prisión Política y Tortura (en adelante CNPPT), se implementó entre 2003 y 2005. En ese marco, diversos profesionales se desempeñaron como mandatarios de la CNPPT para recoger, sistematizar y calificar los testimonios de quienes acudieron a declarar a la Comisión, entre noviembre de 2003 y mayo del 2004. Realizaron, por tanto, una tarea de carácter jurídico consistente en establecer convicción y validez del daño infringido por agentes del Estado durante la dictadura militar a quienes fueron víctimas de dichos abusos. Durante la construcción del primer informe de la CNPPT (2004), dichos profesionales recibieron el testimonio de más de 35.000 personas.

La inquietud de la presente investigación surgió en torno a la experiencia vivida por quienes trabajaron para el primer informe de la CNPPT (2004), recogiendo y sistematizando el testimonio de las víctimas de prisión política y tortura (en adelante PPT). Dichos profesionales llevaron a cabo una labor de carácter pericial, inscrita en una temporalidad previamente determinada y encomendada por el Estado. La pregunta planteada en esta investigación buscó comprender el impacto de la experiencia desarrollada en la Comisión sobre estos profesionales.

\section{Traumatización extrema y trauma psicosocial}

En Chile, los trabajos relativos a los hechos y procesos desencadenados a partir de la dictadura militar, se pueden agrupar en, al menos, tres ámbitos: 1) construcción de memoria: donde se reflexiona en torno al producto y a los procesos de memoria, entretejidos entre lo individual y lo colectivo, a partir del golpe de Estado (Garcés et al., 2000; Manzi, Helsper, Ruiz, Krause \& Krönmuller, 2003; Reyes, 2009; Ruiz \& Krause, 2003, entre otros); 2) traumatiza- ción vicaria y victimización secundaria: en equipos y profesionales que trabajan con temáticas ligadas a la violencia, estudiando lo que les sucede cuando se enfrentan a testimonios de sufrimiento humano (Morales \& Lira, 1997, 2000) y 3) ámbito del impacto y consecuencias traumáticas en las víctimas de las violaciones a sus derechos humanos: donde se han desarrollado importantes conceptualizaciones y modelos de intervención para abordar esas temáticas (Agger \& Sornes Buus, 1996; Castillo, 2009; Castillo \& Morales, 2011; Cornejo et al., 2007; Lira, Becker \& Castillo, 1991; Zapata, López \& Sánchez, 2009).

En este último ámbito, se trabajó el concepto de trauma desde diversas perspectivas. El concepto de traumatización extrema (Becker, Castillo, Gómez, Morales \& Lira, 1988; Bettelheim, 1981; Lira et al., 1991) permite establecer una diferencia entre experiencias traumáticas que son producto de situaciones naturales imprevisibles e involuntarias y situaciones traumáticas predecibles y controladas en el marco de la represión política. Junto con el reconocimiento de la especificidad social del trauma, este concepto alude especialmente a su intencionalidad y a su condición de situación extrema, en el sentido en que busca en forma deliberada la destrucción de la condición de persona y de militante político de quien la recibe.

La noción de trauma psicosocial, propuesta por Ignacio Martín-Baró (1988), surge con la intención de generar un marco conceptual más adecuado para abordar los problemas psicológicos que se derivan de contextos sociohistóricos, integrando lo político en relación directa con la ocurrencia del evento traumático. Desde esta perspectiva, se entiende la situación de tortura como una relación traumatizante entre el Estado o agentes del Estado y personas que fueron sometidas a esta práctica producto de su filiación política o la presunción de ella. Si bien los efectos físicos y psicológicos se manifiestan principalmente en el sujeto sometido, es la sociedad en su conjunto la que resulta impactada, especialmente si se considera que la tortura, junto a las ejecuciones, desapariciones y detenciones, se utilizaron como estrategias de control político para regular el orden social por medio de la impronta de amenaza colecti- 
va y miedo en que vivían los ciudadanos (Lira et al., 1991; Lira \& Castillo, 1991). La sociedad también se traumatiza, en el sentido en que sus relaciones sociales se encuentran perturbadas, lo que se expresa en procesos psicosociales que tienden a la instauración de la desconfianza, la rigidez, el escepticismo y la violencia como forma de resolución de conflictos (Barreto \& Borja, 2007; Morales, 1997).

\section{Comisiones de Verdad y Reparación en Chile}

Las Comisiones de Verdad han existido en varios países en el mundo. Se entienden como organismos temporales, nombrados por una autoridad oficial con un mandato específico, y cuyo objetivo es investigar graves violaciones a los derechos humanos ocurridas en el pasado (Bronkhorst, 2006). Incluyen la elaboración y entrega de un informe público y oficial, que recuerda y trasmite lo sucedido a posteriores generaciones. Estas comisiones comparten un interés historiográfico por establecer una verdad oficial de los hechos ocurridos en el pasado y promover pactos sociales para la convivencia futura (van der Merwe, 2002), orientándose al reconocimiento de la historia "reprimida" o "negada" de la violencia ejercida hacia ciertos grupos sociales (Hamber, 2000).

En Chile, luego de la dictadura de Pinochet, las iniciativas gubernamentales de creación de la Comisión Nacional de Verdad y Reconciliación en 1990, la Mesa de Diálogo en 2001, la Comisión Nacional sobre Prisión Política y Tortura en 2004 y la Comisión Asesora Presidencial para la Calificación de Detenidos Desaparecidos, Ejecutados Políticos y Víctimas de Prisión Política y Tortura en 2009, da cuenta de cómo se han mantenido vigentes los objetivos de verdad y reparación.

En la entrega oficial del Informe de la Comisión de Verdad y Reconciliación en 1991, se generó una versión oficial de la historia de Chile entre 1973 y 1990, estimando en 3.200 las personas que fueron objeto de represión política con causa de muerte, y determinando como violaciones de los derechos humanos no solo actos cometidos por agentes del Estado, sino también otros actos perpetrados por particulares que actuaron bajo pretextos políticos.
Durante 2001, se convoca a diferentes representantes de la sociedad civil, del mundo político, de las iglesias, de las fuerzas armadas y del ámbito de los derechos humanos para establecer una instancia de reencuentro basada en la tolerancia y la transparencia en la denominada Mesa de Diálogo. El objetivo fue establecer acuerdos básicos respecto de los acontecimientos ocurridos en el pasado reciente y esclarecer algunos casos de detenidos desaparecidos y ejecutados políticos.

Luego, en el 2003, en el marco de la propuesta en materia de derechos humanos "No hay mañana sin ayer", se forma la Comisión Nacional sobre Prisión Política y Tortura, con el objetivo de determinar, de acuerdo a ciertos antecedentes, quiénes fueron las personas que sufrieron privación de libertad y torturas por razones políticas, por actos de agentes del Estado o de personas a su servicio, en el período comprendido entre el 11 de septiembre de 1973 y el 10 de marzo de 1990.

En una primera etapa -noviembre de 2003 a mayo de 2004- esta Comisión recibió los testimonios de 35.865 personas, que fueron entrevistadas en su sede en Santiago, en 42 gobernaciones provinciales y en 40 países a través de los consulados y embajadas de Chile en el extranjero. En noviembre de 2004, se dio a conocer públicamente el Informe de la Comisión ${ }^{11}$ que contiene una nómina de las 27.255 personas que la Comisión reconoce como víctimas de privación de libertad y tortura por motivos políticos, producto de las acciones represivas cometidas por agentes e instituciones estatales. En el Informe se encuentra además una explicación de cómo se desarrolló la PPT, así como criterios y propuestas de reparación a las víctimas reconocidas. En una segunda etapa, de noviembre de 2004 a mayo de 2005, la "etapa de reconsideración", la CNPPT recogió nuevos antecedentes de cerca de 8.000 personas que habían declarado pero no calificado en el primer Informe, de los cuales finalmente 1.201 calificaron y accedieron a los beneficios contenidos en la ley, lo que hace un total de 29.201 víctimas de PPT.

1 Disponible en http://www.comisionvalech.gov.cl/ 
La Comisión Asesora Presidencial para la Calificación de Detenidos Desaparecidos, Ejecutados Políticos y Víctimas de Prisión Política y Tortura, elaboró también un informe que fue entregado en agosto de 2011. Este Informe ${ }^{2}$ da cuenta de 622 solicitudes de calificación de detenidos desaparecidos y ejecutados políticos, de los cuales calificaron 30 . También da cuenta de 31.841 nuevas solicitudes de calificación de personas que declararon haber sido víctimas de PPT; de estas solicitudes, fueron calificados 9.795 nuevos casos.

\section{Escuchar la tortura: los profesionales de la CNPPT}

La CNPPT sin duda impactó a las personas que acudieron a declarar ante la Comisión y a sus familias (Riquelme \& Cruz, 2005). Así también, la creación y funcionamiento de la Comisión impactó a la sociedad chilena en su conjunto (Cárdenas et al., 2011).

Las personas que acudieron a declarar a la CNPPT debían completar una ficha especialmente construida para estos fines, respecto de diversos antecedentes y características de su experiencia de PPT. Luego, eran entrevistados por un/a profesional de la Comisión por cerca de una hora, contando su experiencia y entregando antecedentes significativos respecto de esta. El estricto respeto de los límites en la relación con los declarantes y la premisa de no comprometerse con posiciones políticas eran reglas que los profesionales debían acatar a fin de preservar la imparcialidad en el trabajo de la Comisión.

La implementación de la CNPPT instauró un particular fenómeno a partir de su funcionamiento: la figura de los profesionales encargados de recoger los testimonios de PPT, con los consecuentes desafíos profesionales y personales implicados en ese trabajo (Sharim, Kovalskys, Morales \& Cornejo, 2011). Se trató de 50 a 60 profesionales - de distintas edades, profesiones, ámbitos de desempeño y trayectorias de vida- que, desde un posicionamiento particular en el marco de una Comisión instaurada por el Estado, estuvieron en contacto directo con las

2 Disponible en http://www.comisionvalech.gov.cl/InformeComision/Informe2011.pdf historias de las víctimas, recepcionando antecedentes en la Región Metropolitana y en diferentes gobernaciones provinciales a los largo de todo el país.

Intentando comprender este fenómeno, surgió la presente investigación cuyo objetivo fue conocer y comprender el impacto que los profesionales participantes en la CNPPT señalaron haber vivido a partir de esta experiencia.

\section{Método}

El anclaje de esta investigación en el enfoque biográfico y la utilización del relato de vida, se fundamenta en el hecho de que representan un modelo epistemológico y un método de producción de datos, adecuados y coherentes a la problemática de estudio. El enfoque biográfico-narrativo (Cornejo, 2006; Legrand, 1993; Niewiadomski \& De Villers, 2002) articula contribuciones de distintas corrientes de pensamiento en las ciencias sociales (marxismo, existencialismo, estructuralismo, hermenéutica y psicoanálisis), estableciéndose como un enfoque interdisciplinario que incluye influencias diversas. Este enfoque no alude únicamente a una particular metodología de investigación, sino más bien a un enfoque teórico-metodológico que: sostiene una relación articulada entre lo singular y lo social, valoriza la subjetividad como fuente de conocimiento científico, entrega una propuesta compleja acerca de la relación de los individuos con su historia -historia personal, familiar y social-y permite entender la narración como una expresión y construcción de la identidad.

\section{Participantes}

El proceso de producción muestral se inició en mayo de 2005, a partir del contacto con el equipo de profesionales a cargo de la atención de público de la CNPPT. La producción de datos se realizó entre junio y septiembre de 2006, y una segunda etapa entre mayo y septiembre de 2007. En total, se realizaron relatos de vida con 22 profesionales de la CNPPT.

En la producción de datos, se efectuó inicialmente un muestreo teórico (Glaser \& Strauss, 
1967), dando luego paso a un muestreo intencionado (Flick, 2004), ampliando el espectro de participantes ya no solo a profesionales que recepcionaron directamente testimonios en la Comisión, sino también a profesionales que participaron en el procesamiento de datos, el apoyo al proceso de calificación (por ejemplo, la elaboración de informes), así como a aquellos que trabajaron en el equipo central de la Vicepresidencia Ejecutiva, los Comisionados.

De los 22 participantes, en cuanto a su función desempeñada en la CNPPT, 13 trabajaron directamente en la recepción de testimonios: nueve, principalmente en Santiago: dos, en la zona norte y dos en la zona sur del país. Seis de ellos no recibieron testimonios presencialmente, ocupándose del procesamiento de información y la elaboración de bases datos, además de apoyar la calificación, todas actividades realizadas en Santiago.

Respecto a sus profesiones, eran abogados, psicólogos, asistentes sociales y sociólogos. Sus edades oscilaban entre los 20 y 60 años, con un promedio de 41.6 años al momento de la producción de datos. Todos pertenecen predominantemente a sectores socioeconómicos medios.

\section{Diseño}

En la producción de relatos de vida, se realizaron tres encuentros con cada participante, con una duración promedio de una hora y media cada uno. Los intervalos entre cada encuentro oscilaron entre 13 días y dos meses, con un promedio de un mes.

Los relatos de vida fueron grabados en su totalidad, previo consentimiento explícito por parte de los participantes. Entre cada encuentro le fue entregado al participante una transcripción completa de su relato de manera que pudiera leerla y volver sobre ella en el encuentro siguiente.

Los relatos fueron recogidos en su totalidad por psicólogos clínicos (cinco mujeres y dos hombres) con formación teórica y entrenamiento en el enfoque biográfico. La consigna inicial de todos los relatos fue abierta: "cuéntame la historia de tu participación en la CNPPT", para permitir una narración construida por el propio participante.

\section{Procedimiento}

El dispositivo de relatos de vida propuesto a los participantes estipuló su adhesión libre y sin presión de ningún tipo. En este sentido, el hecho que la CNPPT hubiera finalizado sus funciones, no contempló ningún compromiso adicional con esta instancia, lo que quedó claramente consignado. Con cada participante se realizó un proceso de consentimiento informado, firmándose una carta que detalla aspectos referidos a las consideraciones éticas de la investigación, especialmente lo relativo a la confidencialidad y anonimato y a su derecho, en todo el proceso de producción de los relatos, a suspender su participación en el estudio.

Se estimó que el trabajo sobre la propia historia asociada a la CNPPT pudiera hacer emerger dificultades emocionales latentes, por lo que se pensó en posibilidades de derivación para una ayuda profesional para los participantes que así lo requirieran. Esta instancia no fue solicitada por ninguno de los participantes.

Para incluir la subjetividad de la interacción entre quien cuenta su historia y quien la escucha (Legrand, 1999), y dada la necesidad de instancias de triangulación en estudios de tipo cualitativo (Moran-Ellis et al., 2006) que otorguen mayor espesor analítico y mayor complejidad comprensiva a los resultados, se constituyó un dispositivo de interanálisis (Cornejo, Besoaín \& Mendoza, 2011; Cornejo, Mendoza \& Rojas, 2008): cada investigador fue acompañado por otro integrante del equipo de investigación en apoyo a la producción y análisis de los relatos.

\section{Dispositivo de análisis de datos}

Dentro del enfoque biográfico, diversos autores señalan que no existe un método único para el análisis de los datos (Kornblit, 2004), sino que estos se definen en consideración de los objetivos de la investigación, del fenómeno estudiado y de ciertas consideraciones epistemológicas y metodológicas acerca de la construcción de conocimiento científico, planteando una diversidad de posibilidades (Arias, González \& Hernández, 2009; Cornejo et al., 2008). 
Dos lógicas articuladas entre sí fueron implementadas en el trabajo de análisis de los relatos (Cornejo et al., 2008). Una primera lógica singular, se orientó a reconstruir una historia de cada participante a partir de los relatos recogidos. Los métodos de análisis utilizados fueron un ordenamiento cronológico y por eventos (de Villers, 1996) y luego un análisis orientado en lo fundamental por el modelo de la "grounded theory" (Glaser \& Strauss, 1967; Strauss \& Corbin, 1990), que establece un acercamiento sistemático a los datos, articulando un trabajo empírico con un trabajo teórico. La segunda lógica fue transversal, y se construyó con base en la emergencia de ejes analíticos temáticos a partir de los cuales se volvieron a analizar las historias reconstruidas, esta vez intentando rescatar elementos que se orientaran a dar cuenta del fenómeno estudiado.

\section{Resultados}

El presente estudio se orientó a comprender el impacto, los sentidos y las significaciones que los profesionales que participaron en la CNPPT dieron a esta experiencia. Para comprender cómo fue vivenciado este impacto y cómo los profesionales lo significaron, los análisis de sus relatos de vida permitieron construir ciertas dimensiones en base a las cuales fue posible desagregar este impacto.

\section{El impacto emocional de la participación en la CNPPT}

Para las víctimas de tortura, el recuerdo de la experiencia genera vivencias de difícil representación, produciendo un estado de perturbación. Depositar el padecimiento en un contexto relacional permite revertir la imposibilidad de poner palabras, de encontrar significados y de crear tejido psíquico. La experiencia de los profesionales como receptores de testimonios de tortura constituye un evento emocional potencialmente desestabilizador que sigue un curso particular en cada sujeto.

A través de las historias de los profesionales fue posible identificar un conjunto de emociones que se reiteran sistemáticamente y en cuya manifesta- ción se superponen el recuerdo de lo vivido por los declarantes a la Comisión y la experiencia propia de los profesionales en la Comisión. Al recordar retrospectivamente la experiencia testimonial, los profesionales coinciden en la desazón que experimentaron para comprender y tolerar la dimensión traumática de los testimonios; sus historias dan cuenta de emociones y sentimientos de carácter perturbador y displacentero, y también esperanzador y gratificante. Se señalaron emociones asociadas con el miedo y la angustia, con sentimientos de temor, de fragilidad e indefensión. Se refirió también miedo a lo desconocido, donde el recuerdo del temor es reconocido con extrañeza.

(...) las cosas que leí, las cosas que escuché, no podría pensar que hayan seres humanos así y creo que me daba susto... (Antonia, C 148) ${ }^{3}$

Otro tipo de emociones está ligado a los sentimientos relativos a la culpa y la incertidumbre frente al desempeño profesional y a la constatación de cierta pérdida de la capacidad de asombro frente a lo siniestro en los testimonios escuchados, sin lograr reconocer, en esta paradoja, los mecanismos de defensa y su carácter transitoriamente protector.

(...) terminé por ponerme una coraza, tratar de implicarme menos, era triste pero se convertía en una cosa mecánica... (Diana, B 48)

Al cerrar la Comisión, los profesionales relatan sentimientos ligados a la melancolía, en el sentido de rescatar la dimensión gratificante de la experiencia traumática compartida con los testimoniantes, y también sentimientos de rabia y frustración por quedar alejados del trabajo en torno a estas temáticas.

(...) esa fue una participación social que se extingue después de cierto tiempo, dejando la nostalgia de lo

3 Se incluirán viñetas correspondientes a citas textuales extraídas de los relatos de vida de los participantes. La letra señala el orden en la secuencia de relatos $(\mathrm{A}, \mathrm{B}$ o $\mathrm{C}$ ) y el número corresponde al párrafo en la trascripción. Los nombres de los profesionales son de ficción. 
perdido, fue un período lleno de vida y de luz y los más dolorosos también. (Carola, B 488)

(...) después de una entrega tan apasionada me sentí abandonada... (Clara, B 162)

Es posible reconocer en todos los profesionales que el hilo discursivo en sus relatos presenta un desplazamiento sostenido desde sentimientos asociados a lo doloroso hacia otros de carácter alentador vinculados a la satisfacción, el orgullo y el alivio por la tarea.

(...) si tú tienes la vivencia de escuchar tantas cosas, cómo no te va a cambiar la perspectiva de la vida, de los valores... (Isabel, C 234)

(...) de todos mis trabajos quizás ha sido uno de los más bonitos, ser reconocida como una persona que ayudó. (Emma, A 327)

\section{El lugar de la experiencia de la CNPPT en la biografía y en la narración}

En la historia que los profesionales contaron, fue posible identificar hilos conductores que se van entramando a través de la narración y que van dando sentido y significado a la historia relatada, visibilizando la forma que adquirió en cada biografía la experiencia de trabajo en la CNPPT.

La escucha de experiencias traumáticas generó en los profesionales un impacto del que los relatos dan cuenta de distintas maneras, ya que los movilizó a dar sentido a las resonancias personales que esta escucha fue despertando. Dicha experiencia en la Comisión se fue encadenando a hitos biográficos propios, ligados al tema de la violación a los derechos humanos para algunos profesionales, y para otros a vivencias personales de distinto tipo. En ambos casos, estas vivencias los hicieron cuestionar esta particular experiencia de trabajo. En algunos profesionales de manera más visible, y en otros de un modo más encubierto, la experiencia en la CNPPT dio lugar al inicio de un proceso elaborativo que permitiera la integración de dicha experiencia a su trayectoria vital. Desde esta perspectiva, se observan particularidades en relación con el espacio que el trabajo en la Comisión representa en la línea de vida de cada profesional y el modo en que se articula con su biografía.

\section{Una experiencia ambivalente}

Para algunos profesionales, la experiencia en la CNPPT ha quedado provista de un sentido paradojal que da cuenta, por un lado, del orgullo y la importancia que se le otorga y, por otro, de la frustración y el desencanto, lo que dificulta poder cerrar o integrar completamente esta experiencia al resto de la vida.

(...) creo que le daría espacio a otras personas que a lo mejor están más limpias, más... más... con menos sentimientos de culpa... Yo recuerdo que fue una experiencia personal de haberme sentido inútil, y haber sentido una inutilidad total....Una culpa, esa... que pa' mí fue una roca arriba de mí, una roca... (Carola B 479)

\section{Una experiencia desarticulada}

Para otros profesionales, la experiencia en la Comisión parece haber quedado desarticulada, sin conexiones que permitan incorporarla a la trama de la vida. Ya sea porque ha quedado aislada, relegada a un recuerdo en particular, sin significados más globales o articulaciones con otros hitos biográficos, o porque es significada como un intento de cierre, algo que, si bien es posible vincular con experiencias previas, remueve una historia que nunca fue cerrada, por lo que se la deja sin lugar, desvinculada.

(...) La imposibilidad de contarlo todo... tenía una pareja pero estaba más o menos con mi pareja en ese tiempo así que no le contaba nada, no, además que no me escuchaba porque no tenía idea y no quería saber tampoco, así que no... [baja la voz] era lo mismo, si quería hablarle a la pared, entonces... en ese aspecto... entonces no... no le hablaba, y estar solo y callado con eso, me tenía mal. (Pedro, A 94) (...) icómo me deshago de todo esto? No sé, así una sensación que yo tengo de tener medio abierto... ahora es superbueno, porque te permite como que cerrar y además ya puedes decir lo que me llevo 
encima fue lo mejor, ya no llevo encima de mí las historias de las penas de otros, de repente aparecen, claro, pero es distinto, no es la pena que te daba antes. (Diana, C 164-166)

(...) nunca hablamos de lo que nos pasó... no sé por qué, es como tratar de que no reverbere más... en ese momento efectivamente lo tratamos de tapar... (Ricardo B, 98)

\section{Una experiencia articulada}

Algunos profesionales sí parecen haber articulado la experiencia en la CNPPT a otros hitos biográficos, otorgándole significación y un lugar en sus propias historias. En algunos, por ejemplo, esta vivencia les permitió orientar a futuro sus elecciones, en especial en un interés por trabajar en la temática de derechos humanos. Para otros, esta experiencia permitió reafirmar la elección vocacional previa, la que adquiere un nuevo sentido y un mayor grado de compromiso.

Del mismo modo, hay profesionales para los cuales esta experiencia permitió comprender su historia personal o familiar de otra manera y, desde ahí, dar un sentido a la experiencia presente y resignificar parte de la historia pasada. Ligado a lo anterior, para algunos, especialmente para los más jóvenes, esta experiencia generó la posibilidad de un crecimiento y madurez personal, mientras que para los que contaban con más experiencia en el tema, fue una consecuencia natural de su trayectoria laboral y vital.

(...) yo creo que [la Comisión] me ha hecho entender un montón a mis papás. Me ha hecho entender un montón a estos viejos de esta generación (...) de por qué se pusieron así... (Graciela, B 44)

(...) La Comisión fue muy importante en mi vida, o sea yo miro hacia atrás y me doy cuenta que claramente hay un quiebre, en la manera de mirar la vida desde mi trabajo en la Comisión hasta ahora. (Andrés, A 8)

(...) Yo sentía que estaba ocupando un lugar que Dios me había convocado, o sea, yo no soy fanática, ni me doy con una roca en el pecho ni mucho menos, pero yo siento que muchas veces uno cae en el lugar donde corresponde, y como yo soy creyente, siento que tiene que ver con misiones, tiene que ver con aportes, y en ese sentido, a mí me generaba cierta calma también eso. (Camila, B 64)

\section{Una experiencia totalizante}

Finalmente, la experiencia de participación en la CNPPT tomó para algunos profesionales un carácter totalizante, en el sentido de que una parte importante de su vida cobró sentido a raíz de esta experiencia de trabajo. Para algunos, el carácter protagónico de esta vivencia estuvo dado por la intensidad de las emociones que despertó y que están presentes hasta la actualidad. Para otros, de un modo más racional, esta experiencia de trabajo permitió resignificar parte de la vida, y dar así sentido a la trayectoria que los llevó a participar de la Comisión, así como a las decisiones para el futuro.

(...) Fue uno de los momentos en que yo sentía que, el dolor me superó, o sea que no fui capaz racionalmente de elaborar la información que tenía porque no, no pude entender, me quedó grande. (Matilde, A 220)

(...) Caminaba como extraña, que yo sentía que era una sensación extraña como un extraterrestre, como que llevaba una historia y que los demás, como una historia media secreta y los demás en la calle compraban, fumaban, compraban los diarios, como que la vida iba en otra velocidad... (Pilar, B 48)

\section{La institucionalidad del rol de escucha}

Como organización, la CNPPT implicó la articulación de procesos, funciones y sistemas de roles que tuvieron como misión lograr una determinada producción pericial, y al mismo tiempo, la producción discursiva por medio de su Informe. Esta doble función implicó el cruce de roles, propios de la organización CNPPT con otras institucionalidades de la biografía de los profesionales; en este cruce es posible distinguir importantes aspectos relativos al impacto de esta experiencia. Si bien todos los profesionales ampliaron sus funciones, existió una diferencia sustantiva marcada por el hecho de re- 
cibir testimonios directos de tortura, pues los situó en contacto o reencuentro con la historia del país, lo que generó un compromiso emocional con las víctimas de PPT.

(...) Porque era una patita de la reparación... el primer paso, entonces yo quería que también fuera una buena entrevista, o sea, quería que fueran cuidados y que estuvieran bien... (Alicia, B 73)

Quienes realizaron funciones sin contacto directo con los declarantes, hablan de un compromiso, pero que es del orden institucional con la historia del país.

(...) Tengo [recortes de diario de la época de la Comisión] guardados en mi casa, todo en una carpetita, pensando que yo el día de mañana les voy a enseñar a algunos jóvenes, como te digo, futuros profesionales, en que consistió todo este proceso histórico. (Andrés, A 144)

(...) yo estaba todo el rato superconcientizada que el trabajo era de una nobleza superfuerte, que era un trabajo, poderoso, relevante, que estábamos dignificando a las personas que estábamos dándoles un lugar en el discurso, estábamos dándole un nombre de víctima, estábamos ofreciéndoles una oreja oficial, o sea habían muchas cosas cruzadas. (Camila, A 146)

Los profesionales comparten un sentimiento común de legitimidad del rol que describen haber desempeñado en la Comisión, percibiéndose con un buen desempeño. En este sentido, es llamativo que no se observe autocrítica, sino que esta se manifiesta referida a la institución.

(...) Quedé con amargura al escuchar las medidas de reparación... sentí que fue un fraude en realidad... a momentos perdió sentido lo que habíamos hecho. (Facundo, A 27)

Las referencias a la grupalidad, al "nosotros" como soporte en la labor desarrollada, están presentes explícitamente en los profesionales que trabajaron en la Región Metropolitana, en el denominado equipo central. En el caso de profesionales que trabajaron en regiones, la apelación al "nosotros" se hace desde la carencia de este, en el sentido de la percepción de ausencia de un soporte colectivo.

En relación con la institución, son factores comunes la legitimidad de la participación en la CNPPT, fundamentada en historias institucionales previas (familia, trabajo en derechos humanos o posturas en relación a estos en subgrupos socioculturales o políticos), y el orgullo por ser parte de esta institución. También está la decepción y la ambivalencia en relación con la institución, o más bien a su rol pericial.

Los profesionales más jóvenes tienden a conflictuarse en la interacción con sus familias de origen, encontrando dificultades para compartir la experiencia en la CNPPT. Los mayores la significan de un modo más continuo y coherente, concurriendo subcultura y/o tradiciones políticas de izquierda, y la noción de legado en el sentido de responsabilidad histórica. De alguna manera, sienten estar realizando una labor que no solo les pertenece a ellos sino a un colectivo que remite a su historia previa a la participación en la Comisión.

Todos los profesionales parecen muy motivados con el trabajo en la CNPPT y hay diferencias relevantes en cómo llegaron a trabajar en ella. En el caso de los más jóvenes, el trabajo en la Comisión aparece como una oportunidad laboral, pero luego de insertarse se hace un imperativo justificar su rol en la CNPPT de un modo retrospectivo, y al mismo tiempo apelando a sus historias familiares (padres) para dar cuenta de su propio lugar en relación al tema de la tortura:

(...) Con la poca posibilidad que nuestra generación tenía, ese aporte era gigante al lado de lo que podía no haber hecho. Entonces qué bueno que yo sí tuve la posibilidad de cumplir un rol histórico... los más jóvenes nos sentíamos superorgullosos y como, bueno aparte medios sorprendidos, pero con una sensación como de responsabilidad de misión... era una cosa bien fraterna como de compañeros haciendo algo por la patria. (Diana, B 42)

De algún modo, todos los profesionales intentan legitimar la opción de trabajar en la temática de 
PPT, ya sea desde la recuperación de la continuidad histórica, biográfica y política, o la historia familiar.

Los profesionales, de manera predominante y bastante homogénea, señalan haberse conmovido y haber empatizado con las personas que declararon en la Comisión, llegando a ciertos grados de identificación con los declarantes, buscando contenerlos y apoyarlos, y teniendo la noción de un rol histórico que anclan en distintos lugares sociales y temporales.

(...) Yo sabía que era importante, pero después caché que era histórico, o sea histórico para la sociedad, histórico para el país, para el mundo, pero sobre todo para mí... También como que la experiencia de otros se transforma en la [remarcando esta palabra] experiencia de ambos, que no pasa a ser de él ni mía, sino del conjunto. (Alicia, B 33)

Resulta fundamental respecto a la institucionalidad del rol en la CNPPT, una clara frontera entre el proceso y la noción ciudadana de haber sido miembro de la Comisión. Así, luego de experimentar gran orgullo por la pertenencia al rol del Estado reparador, los profesionales denotan un cierto grado de marginalidad social al terminar la participación, tanto por disolución de la institución CNPPT como por la percepción mayoritaria de las políticas reparatorias como deficitarias, y la escasa gravitación social que perciben de parte de la sociedad chilena hacia el tema de la tortura.

\section{Discusión}

Los resultados permiten distinguir ciertas dimensiones -emocional, biográfica-narrativa, e institucional-, entendidas como coordenadas por las que circulan los procesos elaborativos que resultaron del impacto de la labor pericial/reparatoria desarrollada por los profesionales de la CNPPT. El hablar de coordenadas apunta, más que a entender los procesos elaborativos dando lugar a un producto, promoviendo un proceso que circula por rutas posibles, por espacios biográficos (al decir de Arfuch, 2002) y conjugando la historia personal y la historia social de los profesionales de la CNPPT, revelándolas como profundamente articuladas y entrelazadas entre sí.

Para los profesionales, la experiencia de escuchar testimonios de prisión y tortura en la Comisión tuvo un primer efecto que ocurre a nivel de un impacto emocional, en el sentido de experiencias emocionales disruptivas y eventualmente traumáticas que invaden y demandan una significación. Parte de esta significación se construye con el relato de la historia de participación en la Comisión, dándole un lugar en la narración y la biografía, lugares diferentes según los profesionales y que apuntan a las diferentes formas en que fueron impactados por lo traumático de los testimonios escuchados y por la forma en que esta experiencia adquiere un lugar en su biografía. Sin embargo, la experiencia en la Comisión se enmarca además en una historia social: la de un país que luego de un daño infringido por parte de agentes del Estado, intenta construir una verdad sobre lo ocurrido y reparar el daño. Se instala así una institucionalidad en el rol de escucha, la que es cuestionada y otorga, al ser relatada, significaciones colectivas a esta experiencia.

\section{Impacto emocional y re- construcción biográfica}

Al escuchar la experiencia de tortura de los declarantes en la CNPPT, surgieron emociones disruptivas en los profesionales con la tendencia a ponerse en espejo con las emociones del sujeto violentado, en el sentido en que la declaración que este hace de su experiencia de tortura, le es transmitida y, en cierto modo, trasladada al profesional de la Comisión. Esta escucha lo deja insoslayablemente capturado por los contenidos que son escuchados en un vínculo particular, constituyéndose así en una "cadena de escucha" (Cornejo, Rojas \& Mendoza, 2009; Cornejo, Brackelaire \& Mendoza, 2009), cadena que enlaza a quien escucha sobre la tortura y se extiende desde los declarantes a los profesionales de la Comisión, y desde estos a los investigadores que solicitaron sus relatos en el marco de esta investigación.

En este sentido, la necesaria implicación emocional en la relación establecida por parte de los 
profesionales con los declarantes -en la medida en que fue sostenida en el tiempo de duración del funcionamiento de la Comisión-, generó no solo el desgaste habitual del trabajo con el sufrimiento humano (Morales, Pérez \& Menares, 2003), sino además la necesidad de hacer una lectura retrospectiva que fue re-construyendo y re-significando la biografía de los profesionales, dado que sus propias historias personales y sociales resonaron con esta escucha y fueron sirviendo de telón de fondo para continuar con la escucha, escucha cargada de horrores, de sufrimientos y de dolores.

La ambivalencia de la escucha y la institución de la ley

Si bien existe una gradiente de impacto diferencial en función del rol (recepción directa de testimonios $\mathrm{u}$ otras labores como procesamiento de la información o elaboración de informes) y del soporte institucional de la organización de la CNPPT, desarrollar distintos funciones se tradujo en experiencias distintas que se engarzaron con las particulares biografías personales, familiares y sociales de los profesionales.

Así, el rol de los profesionales de la Comisión puede entenderse desde ciertas ambivalencias en tanto rol de escuchas del Estado (Morales \& Cornejo, 2012), ambivalencias que se anclan, se ligan y se articulan en la biografía familiar de cada profesional, para configurar -o por la necesidad de configurar- un legado. Este legado se instala como la necesidad de integrar el rol ejercido y la política pública de verdad y reparación que emanó de este, con la propia biografía inscrita y puesta en tensión ya no solo como sujeto individual y privado, sino como sujeto social y público, como ciudadano inscrito en la historia social de un país.

Al significarse los profesionales de la CNPPT como sujetos sociales, la ambivalencia de la escucha se hace en espejo con la de la institución de la ley a partir del trabajo de la Comisión. Se instituye una ley que no lo es: se registran crímenes pero no se sancionan, sino que se generan medidas de reparación hacia quienes fueron víctimas, sin acciones punitivas para quienes fueron victimarios ${ }^{4}$. Así, la justicia es una deuda contraída y no trasladable a otros, lo que nuevamente remite al legado de los profesionales como sujetos sociales, en el sentido de haber sido testigo de un crimen pero no se puede atestiguar para la sanción del mismo.

\section{La "Escucha del Estado"}

La figura de los profesionales como habiéndose constituido en "escuchas del Estado" en la Comisión (Buzzoni, 2008; Cornejo, Rojas \& Mendoza, 2009), los ubicó en una posición compleja, que requirió simultáneamente de un compromiso emocional y de uno institucional, este último materializado en el mandato de la Comisión. Este mandato implicaba recopilar antecedentes y generar la convicción de veracidad, coherencia y consistencia de la declaración de las personas que acudieron a la Comisión, pero al estar determinado por lo traumático-dados los contenidos de las declaraciones- resultó difícil sostener la escucha únicamente desde este lugar institucional.

La compleja capacidad de escuchar y la consecuente movilización emocional que desafió a los profesionales de la Comisión, puede comprenderse apelando al concepto de "desasosiego empático" de LaCapra (2005) que permite explicar la posición que los investigadores adoptan al intentar acceder a contenidos relativos a experiencias traumáticas de carácter sociopolítico. Esto genera inevitablemente en quien escucha vivencias emocionales de molestia e incomodidad. El desasosiego empático sería un requisito esencial para crear una posición de escucha que permita construir un contexto relacional veraz y confiable para quien relata sus experiencias.

Es posible pensar entonces que el desosiego empático habría permitido a los profesionales de

4 Las medidas de reparación para las personas que calificaron en la CNPPT, consisten en beneficios económicos: pensión mensual, ayuda para estudios superiores, beneficios en atención de salud física y salud mental en el sistema de la red asistencial de salud pública, beneficios habitacionales y exención del servicio militar obligatorio. Respecto a los victimarios, cabe señalar que de acuerdo a la ley instituida a partir del trabajo de la Comisión, se dejó establecido por 50 años el secreto de las declaraciones recibidas en la Comisión. 
la CNPPT asumir su rol de escuchas del Estado, evitando la polarización entre una total identificación con los declarantes o bien tomando una objetividad distante, facilitando la construcción de un vínculo que apela a la contención del otro, disposición imprescindible para la expresión y atención a eventos y vivencias del pasado cargadas con lo traumático (Castillo \& Morales, 2011; Lira et al., 1991).

En este sentido, la escucha de los declarantes de la Comisión ubicó a los profesionales en un quehacer articulatorio del recuerdo de la experiencia traumática, que inevitablemente está mediado por la culpa ante la frustración de ejercer un rol solo pericial, que promueve la necesidad de reparación tanto desde el sujeto como ciudadano, como portador de una política de Estado. Para elaborar aquello, pareciera que el soporte institucional otorgado por la organización de la Comisión fue percibido como insuficiente, no necesariamente porque no hubo instancias de capacitación y de autocuidado, sino porque, durante y sobretodo al terminar el trabajo de la Comisión, los profesionales señalan haber escuchado "un secreto" (Cornejo, Rojas \& Mendoza, 2009), secreto asociado a la experiencia traumática específica del horror de la tortura. Este particular secreto puede ser entendido como un secreto público, en el sentido de que si bien el Informe de la CNPPT es público y da cuenta de modalidades y lugares de detención y tortura e identifica a quienes declararon en la Comisión y fueron calificados, no da cuenta de las experiencias específicas que cada profesional escuchó, respecto de las cuales tuvo que mantener reserva. En este sentido, surge la dificultad de cerrar dicha experiencia una vez concluido el trabajo de la Comisión, sin poder hacer el duelo, significar fácilmente esta experiencia, ponerla en palabras, quedando atrapada en las palabras del dolor escuchado. Esto probablemente motivó a los profesionales a participar de manera comprometida en esta investigación, habiendo señalado la importancia que tuvo para ellos el ejercicio propuesto en los relatos de vida al contar su historia de participación en la Comisión, poniendo palabras, significados y sentidos a esta experiencia.

\section{Referencias}

Agger, I. \& Sornes Buus, J. (1996). Trauma y cura en situaciones de terrorismo de Estado. Santiago: Ediciones Chile/América CESOC.

Arfuch, L. (2002). El espacio biográfico. Dilemas de la subjetividad contemporánea. Buenos Aires: Fondo de Cultura Económica.

Arias, V., González, L. \& Hernández, N. (2009). Constitución de sujeto político: historias de vida política de mujeres líderes afrocolombianas. Universitas Psychologica, 3(8), 639-652.

Barreto, I. \& Borja, H. (2007). Violencia política: algunas consideraciones desde la psicología social. Revista Diversitas - Perspectivas en Psicología, 3(1), 109-119.

Becker, D., Castillo, M. I., Gómez, E., Kovalskys, J., \& Lira E. (1990). Psicopatologíay proceso psicoterapéutico de situaciones políticas traumáticas. En I. Martín-Baró (Ed.), Psicología social de la guerra: trauma y terapia (3ํe․, pp. 46-52). San Salvador: UCA Editores.

Bettelheim, B. (1981). Sobrevivir. El holocausto una generación después. Barcelona: Grijalbo.

Bronkhorst, D. (2006). Truth and justice: A guide to truth commissions and transitional justice. Amsterdam: Amnesty International.

Buzzoni, M. E. (2008). Escuchar desde el Estado: significados asociados a la relación establecida entre los profesionales y las personas que concurrieron a declarar a la Comisión Nacional sobre Prisión Política y Tortura. Tesis no publicada para la obtención del título de Magíster en Psicología Clínica, Escuela de Psicología, Pontificia Universidad Católica de Chile, Chile.

Cárdenas, M., Páez, D., Bilbao, M., Arnoso, M., Asún, D. \& Fernández, G. (2011). Collective processes, social and psychological well-being: The psychosocial impact of the Chilean "Truth and Reconciliation" and "Political Imprisonment and Torture" commissions. Manuscrito presentado para su publicación.

Castillo, M. -I. (2009). Transmisión de la violencia y memoria social. Revista Praxis, 15, 99-114.

Castillo, M. -I. \& Morales, G. (2011). Psicoterapia grupal y tortura. En G. Morales, B. Ortúzar \& E. Thumala 
(Eds.), Psicoterapia psicoanalítica de grupo y vínculos (pp. 171-189). Santiago: Orjikh Ediciones.

Comisión Nacional sobre Prisión Política y Tortura. (2004). Chile. Informe de la Comisión. Disponible en http://www.comisiontortura.cl/inicio/index.php

Cornejo, M. (2006). El enfoque biográfico: trayectorias, desarrollos teóricos y perspectivas. Psykhe, 15(1), 95-106.

Cornejo, M., Besoaín, C. \& Mendoza, F. (2011). Desafíos en la generación de conocimiento en la investigación social cualitativa contemporánea. Forum: Qualitative Social Research, 12(1), Art. 9. Disponible en http://nbnresolving.de/urn:nbn:de:0114fqs110196

Cornejo, M., Brackelaire, J. -L. \& Mendoza, F. (2009). Des chaînes du silence à la chaîne de l'écoute. Une recherche à partir des récits des professionnels de la Commission Nationale sur l'emprisonnement politique et la torture au Chili. Cahiers de Psychologie Clinique, 32(1), 205-231.

Cornejo, M., Mendoza, F. \& Rojas, R. C. (2008). La investigación con relatos de vida: pistas y opciones del diseño metodológico. Psykhe, 17(1), 29-39.

Cornejo, M., Rojas, R. C., Buzonni, M. E., Mendoza, F., Concha, M. \& Cabach, C. (2007). Prisión política y tortura: desde las intervenciones psicosociales a las políticas de reparación. Persona y Sociedad, 21(1), 58-81.

Cornejo, M., Rojas, R. \& Mendoza, F. (2009). From testimony to life story: The experience of the professionals of the Chilean National Commission on Political Imprisonment and Torture. Peace \& Conflict, Journal of Peace Psychology, 15(2), 111-133.

de Villers, G. (1996). Le récit de vie comme approche de recherche-formation. In D. Desmarais \& J. M. Pilon (Coords.), Pratique des histoires de vie. Au carrefour de la formation, de la recherche et de l'intervention (pp. 107-134). París: L'Harmattan.

Flick, U. (2004). Introducción a la investigación cualitativa. Madrid: Ediciones Morata.

Garcés, M., Milos, P., Olguín, M., Pinto, J., Rojas M. T. \& Urrutia, M. (2000). Memoria para un nuevo siglo. Chile, miradas a la segunda mitad del siglo XX. Santiago: LOM Ediciones.
Glaser, B. \& Strauss, A. (1967). The discovery of grounded theory: Strategies for qualitative research. New York: Aldine Publishing Company.

Hamber, B. (2000). Repairing the irreparable: Dealing with double-binds of making reparations for crimes of the past. Ethnicity and Health, 5(3-4), 215-226.

Kornblit, A. L. (2004). Historias y relatos de vida: una herramienta clave en metodologías cualitativas. In A. L. Kornblit (Coord.), Metodologías cualitativas en ciencias sociales (pp. 15-33). Buenos Aires: Editorial Bilblos.

LaCapra, D. (2005). Escribir la historia, escribir el trauma. Buenos Aires: Nueva Visión.

Legrand, M. (1993). L'approche biographique. Paris: Desclée de Brouwer.

Legrand, M. (1999). La contra-transferencia del investigador en los relatos de vida. Proposiciones, 29, 115-121.

Lira, E., Becker, D. \& Castillo, M. I. (1991). Derechos humanos: todo es según el dolor con que se mira. Santiago, Chile: ILAS.

Lira, E. \& Castillo, M. I. (1991). Psicología de la amenaza política y del miedo. Santiago: Instituto Latinoamericano de Salud Mental y Derechos Humanos/ Ediciones Chileamérica, CESOC.

Manzi, J., Helsper, E., Ruiz, S., Krause, M. \& Kronmuller, E. (2003). El pasado que nos pesa: la memoria colectiva del 11 de septiembre de 1973. Revista de Ciencia Política, 23(2), 177-214.

Martín-Baró, I. (1988). La violencia política y la guerra como causa del trauma psicosocial. Revista de Psicología de El Salvador, 7(28), 123-141.

Morales, G. (1997). Subjetividad, psicología social y problemas sociales. Revista de Psicología, 7(1), 27-34.

Morales, G. \& Cornejo, M. (2012). L'ambivalence du travail d'écoutant de la torture. Le cas du Chili. Manuscrito presentado para su publicación.

Morales, G. \& Lira, E. (1997). Dinámicas de riesgo y cuidado de equipos que trabajan con situaciones de violencia. En E. Lira (Ed.), Reparación, derechos humanos y salud mental (pp. 105-121). Santiago: CESOC.

Morales, G. \& Lira, E. (2000). La "receta del autocuidado": los riesgos de equipo de programas que trabajan con situaciones de violencia. En Sociedad Chilena de Psicología Clínica (Eds.), Violencia en 
la cultura: riesgos y estrategias de intervención (pp. 55-80). Santiago: Ediciones Sociedad Chilena de Psicología Clínica.

Morales, G., Pérez, C. \& Menares, A. (2003). Procesos emocionales de autocuidado y riesgos de equipo en profesionales que trabajan con el sufrimiento humano. Revista de Psicología, 12(1), 9-25.

Moran-Ellis, J., Alexander, V., Cronin, A., Dickinson, M., Fielding, J., Sleney, J., et al. (2006). Triangulation and integration: Processes, claims and implications. Qualitative Research, 6(19), 45-59.

Niewiadomski, C. \& de Villers, G. (2002). Prolégomènes. In C. Niewiadomski \& G. de Villers (sous la direction de), Souci et soin de soi. Liens et frontières entre histoire de vie, psychothérapie et psychanalyse (pp. 11-46). Paris: L'Harmattan.

Reyes, M. -J. (2009). Generaciones y memoria: una dialógica conflictiva. Revista Praxis, 15, 77-97.

Riquelme, J. \& Cruz, G. (2005). Impacto de la Comisión Nacional sobre Prisión Política y Tortura en Chile. RevistaVirtu@l ILAS, 4. Recuperado el 17 de octubre de 2006, de http://www.ilas.cl/revi_4.html
Ruiz, M. S. \& Krause, M. (2003). Memorias colectivas y reconciliación nacional en Chile: a treinta años del golpe militar. Persona y Sociedad, 12(3), 221-248.

Sharim, D., Kovalskys, J., Morales, G. \& Cornejo, M. (2011). Trauma psicosocial y memoria: diseño de un dispositivo biográfico para investigar el impacto de la Comisión de Prisión Política y Tortura en Chile. Revista Estudios Sociales, 40 (agosto), 81-88.

Strauss, A. \& Corbin, J. (1990). Basics of qualitative research: Grounded theory procedures and techniques. Newbury Park: Sage.

van der Merwe, H. (2002). National narratives versus local truths: The truth and reconciliation commission's engagement with Duduza. In D. Posel \& G. Simpson (Eds.), Commissioning the past: Understanding South Africa's truth and reconciliation commission (pp. 269-281). Johannesburg: Witwatersrand University Press.

Zapata, P., López, F. \& Sánchez, M. (2009). Concepción de mundo, aspectos contextuales y bienestar psicológico en chilenos supervivientes a experiencias de prisión y tortura por motivos políticos a más de 30 años de ocurridos los hechos. Universitas Psychologica, 3(8), 761-769. 